\title{
Regional suicide prevention planning: a dynamic simulation modelling analysis
}

\author{
Adam Skinner, Jo-An Occhipinti, Yun Ju Christine Song and Ian B. Hickie
}

\section{Background}

Regional planning may help to ensure that the specific measures implemented as part of a national suicide prevention strategy are aligned with the varying needs of local services and communities; however, there are concerns that the reliability of local programme development may be limited in practice.

\section{Aims}

The potential impacts of independent regional planning on the effectiveness of suicide prevention programmes in the Australian state of New South Wales were quantified using a system dynamics model of mental health services provision and suicidal behaviour in each of the state's ten Primary Health Network (PHN) catchments.

\section{Method}

Reductions in projected suicide mortality over the period 20212031 were calculated for scenarios in which combinations of four and five suicide prevention and mental health services interventions (selected from 13 possible interventions) are implemented separately in each PHN catchment. State-level impacts were estimated by summing reductions in projected suicide mortality for each intervention combination across PHN catchments.

\section{Results}

The most effective state-level combinations of four and five interventions prevent, respectively, $20.3 \%$ and $22.9 \%$ of 10312 suicides projected under a business-as-usual scenario (i.e. no new policies or programmes, constant services capacity growth). Projected numbers of suicides under the optimal intervention scenarios for each PHN are up to 6\% lower than corresponding numbers of suicides projected for the optimal state-level intervention combinations.

\section{Conclusions}

Regional suicide prevention planning may contribute to significant reductions in suicide mortality where local health authorities are provided with the necessary resources and tools to support reliable, evidence-based decision-making.

\section{Keywords}

Australia; computer simulation; mental health services; suicide; system dynamics.

\section{Copyright and usage}

(C) The Author(s), 2021. Published by Cambridge University Press on behalf of the Royal College of Psychiatrists. This is an Open Access article, distributed under the terms of the creative Commons Attribution licence (http://creativecommons.org/ licenses/by/4.0/), which permits unrestricted re-use, distribution, and reproduction in any medium, provided the original work is properly cited.
Preventing suicide is a global public health priority. More than 800000 people are estimated to have died by suicide in 2016, including nearly 137000 children and young adults aged 10-24 years. Among people aged 15-29 years, suicide is the second leading cause of mortality worldwide, accounting for around $8 \%$ of deaths in this age group every year. ${ }^{2}$ The World Health Organization proposed in its Global Mental Health Action Plan, 2013-2020 that national governments should develop and implement comprehensive suicide prevention strategies with the collective goal of achieving a $10 \%$ decrease in the global suicide rate by $2020 .^{3}$ As of November 2018, 38 countries had adopted a national strategy developed specifically to prevent suicide and intentional self-harm. ${ }^{4}$ Typically, these strategies incorporate a broad range of objectives: restricting access to commonly-used means of suicide, increasing the availability of mental health services for people at risk of suicidal behaviour, providing improved care to people who have selfharmed, promoting responsible reporting of suicide by the media, increasing public awareness of suicide and mental disorders and improving suicide surveillance systems. ${ }^{4}$

National suicide prevention strategies are often implemented locally via (semi-)independent subnational health authorities to accommodate potentially substantial regional variation in the incidence and underlying causes of suicide and intentional self-harm. ${ }^{4}$ Multiple ecological studies have revealed significant heterogeneity in local suicide rates that may be ascribed (at least partially) to geographical variation in social and economic risk-modifying factors, including alcohol consumption, population density, unemployment, poverty and deprivation, and interpersonal violence ${ }^{5-10}$ Partial (non-uniform) remodelling of mental healthcare provision and the dependence of policy intervention effects on local context (catchment population size, case mix and other factors affecting demand for services) may also contribute significantly to regional variation in suicide mortality. ${ }^{6,11,12}$ Nevertheless, while regional planning may help to ensure that the specific programmes and policies implemented as part of a national suicide prevention strategy are aligned with the varying needs of local services and communities, concerns that the reliability of regional decision-making may be limited in practice have prompted a shift toward more centralised suicide prevention planning in some countries. $^{13}$

\section{Objectives}

This paper presents an analysis of the potential impact of independent regional planning on the effectiveness of suicide prevention programmes, focusing on the Australian state of New South Wales (NSW). Using a system dynamics model of mental health services provision and suicidal behaviour in each of the state's ten Primary Health Network (PHN) catchments, we ran a set of simulation analyses designed to address three principal study aims: first, to determine the extent to which significant regional variation in population and health system characteristics (e.g. prevalence of mental disorders, mental health services accessibility and rates of increase in services capacity) modifies the effects of individual suicide prevention measures on local suicide rates; second, to quantify the potential impact of optimal regional planning (i.e. relative to state-level 
planning) on the effectiveness of suicide prevention programmes comprising multiple interventions implemented in parallel; and third, to assess the capacity of suboptimal commissioning decisions to limit (or reverse) any potential benefit of local programme development.

\section{Method}

\section{Context}

NSW is the most populous state in Australia, with an estimated resident population of 8.16 million (as of 31 March 2020), comprising $31.8 \%$ of the total Australian population. ${ }^{14}$ Government-funded suicide prevention services provided in the state are commissioned locally via ten PHNs, each of which supports a geographically defined population of between approximately 243000 and 1.57 million residents (based on 2016 population estimates; see Supplementary Table 1 available at https://doi.org/10.1192/bjo. 2021.989). Population density varies substantially among PHN catchments, from 2512 people per square kilometre for Central and Eastern Sydney PHN to $<1$ person per square kilometre for Western NSW PHN. The distribution of socioeconomic disadvantage across catchments is also highly uneven: $44.2 \%$ of the South Western Sydney PHN catchment population resides in Level 2 Statistical Areas (SA2s) with Index of Relative Socio-Economic Disadvantage scores in the lowest quintile for the state, ${ }^{15}$ whereas the Northern Sydney PHN catchment contains no SA2s with an Index of Relative Socio-Economic Disadvantage score in the lowest quintile (i.e. all residents live in less disadvantaged SA2s). Mean yearly suicide rates for the period 2001-2017 range from 7.61 suicides per $10^{5}$ population for Western Sydney PHN to 12.18 suicides per $10^{5}$ population for North Coast PHN (Supplementary Table 1).

\section{Model structure, outputs and calibration}

The system dynamics model used for the analyses presented here is based on a similar model recently developed for Hunter New England and Central Coast PHN using a participatory modelling approach that involved diverse stakeholders, including representatives from health and social policy agencies, local government, non-government organisations, primary care providers, emergency services, research institutions, community groups and people with lived experience of mental illness and intentional self-harm. ${ }^{16}$ The core model structure, replicated for each PHN, comprises a set of interconnected sub-models or sectors, including: (a) a population sector, capturing changes in catchment population size resulting from births, migration and mortality; (b) a psychological distress sector that models flows of people to and from states of low psychological distress (Kessler-10 score of 10-15) and moderate to very high psychological distress (Kessler-10 score of 16-50); (c) a mental health services sector, modelling the movement of psychologically distressed patients through a network of possible service pathways involving general practitioners, psychiatrists and allied mental healthcare providers, psychiatric in-patient care, community mental healthcare services and online services and (d) a suicidal behaviour sector that captures numbers of self-harm hospitalisations and suicides. Detailed descriptions of all model sectors are provided in the Supplementary Material. Parameter estimates and other numerical inputs were derived from published research or publicly available data (where possible), or were estimated via constrained optimisation (see below). Model construction and analysis were performed with Stella Architect version 1.9.4 (isee systems, Lebanon, NH, USA; see www.iseesystems.com).
All data used in this study are publicly available, so ethical approval was not required.

Primary model outputs include total (cumulative) numbers of self-harm hospitalisations and suicides (calculated for each PHN catchment and the state as a whole) and numbers of self-harm hospitalisations and suicides per year. The model also provides estimates of the prevalence of moderate to very high psychological distress and a range of services-related outcomes, including numbers of mental health-related emergency department presentations (total and per year), numbers of general practice and community-based specialised mental health services consultations, numbers of psychiatric and acute (general) hospital admissions, hospital and community-based services waiting times, and numbers of patients disengaging from services because of excessive waiting times or dissatisfaction with the care they receive. Outputs are calculated every 0.875 days (the numerical integration time step, $d t$, was set to one-eighth of a week; see Sterman, ${ }^{17}$ Appendix A) over a period of 20 years, starting from 1 January 2011, so that the impacts of suicide prevention and mental health services interventions were modelled from the time of implementation to the start of 2031 (simulations were run from 2011 to permit comparisons of model outputs with historical data; see Supplementary Material). The analyses presented here focus on projected total numbers of suicides for the state and each PHN catchment.

Parameter values that could not be derived directly from available data or published research were estimated via constrained optimisation, implemented in Stella Architect version 1.9.4, using historical time-series data on the prevalence of moderate to very high psychological distress, self-harm hospitalisation and suicide mortality rates, and rates of mental health services usage (numbers of mental health-related emergency department presentations, general practice and community-based mental health services consultations, and psychiatric and general hospital admissions per year). Powell's method ${ }^{18}$ was employed to obtain the set of (optimal) parameter values minimising the mean of the absolute differences between the observed time-series values and the corresponding model outputs, where each difference was expressed as a percentage of the observed value (i.e. the mean absolute per cent error was used as the objective function for the optimisation analyses). ${ }^{17}$

\section{Simulation experiments and sensitivity analyses}

Reductions in projected suicide mortality were calculated for intervention scenarios in which one or more of 13 suicide prevention and mental health services interventions are implemented in a single PHN catchment. Details of all interventions are provided in Supplementary Table 2. Note that only the direct effects of each intervention are specified explicitly in the model; indirect effects are generated automatically by the model structure, so that the total effects (direct and indirect) of separate interventions implemented in parallel are not necessarily additive (i.e. interventions may have synergistic or antagonistic effects when combined). PHN-specific reductions in suicide mortality were calculated for the 10-year period from 1 January 2021 to the start of 2031 by subtracting total (cumulative) numbers of suicides projected under a given intervention scenario from the total number of suicides projected under a baseline scenario (corresponding to business as usual) in which existing suicide prevention policies and services remain in place and mental health services capacity continues to increase at current rates. State-level intervention effects were obtained by summing PHN-specific reductions in projected numbers of suicides across PHN catchments. 
Potential variation in the effects of individual interventions among PHN catchments was assessed by comparing percentage reductions in total numbers of suicides for the same intervention implemented separately in each catchment (study aim 1). We quantified the impact of optimal regional planning on the effectiveness of multi-component suicide prevention programmes comprising four and five interventions (study aim 2), by calculating differences between the maximum reduction in projected numbers of suicides for each PHN catchment (i.e. given the best combination of interventions for the catchment) and $\mathrm{PHN}$-specific reductions in projected numbers of suicides under the optimal state-level intervention scenario (the combination of interventions minimising state-level suicide mortality when implemented in all PHN catchments). Our choice of intervention set sizes (i.e. 4-5) reflects the fact that suicide prevention programmes are generally implemented within resource-constrained settings, where only a limited number of interventions can be supported. The capacity of suboptimal intervention selection to limit any potential benefit of regional planning was assessed for each PHN catchment by determining the number of intervention scenarios preventing fewer suicides than the optimal state-level scenario (study aim 3 ).

Sensitivity analyses were performed to assess the impact of uncertainty in estimates of the direct effects of the interventions on the simulation results. We used Latin hypercube sampling to draw 100 sets of values for selected model parameters determining the direct effects of the interventions on psychological distress, suicidal behaviour and the flow of patients through mental health services from a uniform joint distribution spanning $+/-20 \%$ of the default values (see Supplementary Material). Differences in projected (total) numbers of suicides between the baseline and intervention scenarios were calculated for each set of parameter values and summarised using simple descriptive statistics.

\section{Results}

Figure 1 shows reductions in projected numbers of suicides for scenarios in which the modelled interventions are implemented separately in each PHN catchment. Post-suicide attempt care and social-connectedness programmes both yield more or less uniform reductions in suicide mortality across PHN catchments, preventing, respectively, $6.6-7 \%$ and $4.4-6.1 \%$ of suicides projected under the baseline (i.e. business as usual) scenario. Percentage reductions in projected numbers of suicides for technologyenabled care coordination (3.0-6.7\%) and family psychoeducation and support programmes $(2.7-8.3 \%)$ vary considerably among PHN catchments, while safety planning yields substantially greater percentage reductions in projected suicide mortality in the Western NSW PHN catchment (9.9\%) and Murrumbidgee PHN catchment (8.1\%) than in other PHN catchments (3.0-4.1\%). Psychiatrist and allied health services capacity increases have limited (if any) impact on projected numbers of suicides in the South Eastern NSW PHN, Western NSW PHN, Hunter New England and Central Coast PHN, North Coast PHN and Murrumbidgee PHN catchments (maximum percentage reduction $0.4 \%$ ), but prevent $2.1-4.7 \%$ of projected suicides in the remaining PHN catchments. Awareness campaigns similarly have widely varying impacts on projected suicide mortality across PHN catchments, significantly increasing numbers of suicides (by up to $6.4 \%$ ) in several catchments, while preventing up to $1.4 \%$ of suicides in other catchments.

The optimal state-level combination of four interventions includes post-attempt care, technology-enabled care coordination, family psychoeducation and support programmes, and social-connectedness programmes (Table 1); this intervention combination prevents $20.3 \%$ of 10312 suicides projected under the baseline scenario over the 10-year forecast period (i.e. 1 January 2021 to the start of 2031) and is the best-performing combination of interventions for all PHNs except the Western NSW PHN and Murrumbidgee PHN. A combination of post-attempt care, safety planning, community-based acute care services and social-connectedness programmes yields the greatest reductions in suicide mortality in the Western NSW PHN and Murrumbidgee PHN catchments, preventing, respectively, 6\% (95\% interval 3.5-4.1\%) and 5.2\% (95\% interval $3.2-7.2 \%$ ) of suicides projected under the optimal state-level intervention scenario (Table 1, Fig. 2). The most effective statelevel combination of five interventions, including post-attempt care, safety planning, technology-enabled care coordination, family psychoeducation and support programmes, and social-connectedness programmes, prevents $22.9 \%$ of suicides projected under the business as usual scenario. A combination of postattempt care, technology-enabled care coordination, family psychoeducation and support programmes, awareness campaigns and social-connectedness programmes performs better than the optimal state-level combination in the South Eastern NSW PHN catchment, preventing an additional 16.2 suicides (95\% interval 1.7-27.3) over the forecast period (Table 1 and Fig. 2).

More than $90 \%$ of all possible combinations of four interventions selected from the 13 interventions modelled prevent fewer suicides than the optimal state-level intervention combination in the Western NSW PHN catchment (666 out of 715 possible intervention combinations; 93.1\%) and Murrumbidgee PHN catchment (665 out of 715 combinations; 93\%) (Fig. 3). Only one of the 1287 possible combinations of five interventions (i.e. $0.08 \%$ ) is more effective than the optimal state-level combination in the South Eastern NSW PHN catchment.

\section{Discussion}

The simulation results presented here indicate that while some suicide prevention and mental health services interventions have consistent, relatively large effects on suicidal behaviour (e.g. postsuicide attempt care, social-connectedness programmes), the efficacy of other interventions varies considerably across PHN catchments. Providing safety plans and follow-up care to suicidal patients who present to emergency departments, for example, is substantially more effective in the Western NSW PHN and Murrumbidgee PHN catchments than in other PHN catchments, due primarily to regional differences in per capita emergency department presentation rates (per capita presentation rates are higher in the Western NSW PHN and Murrumbidgee PHN catchments, effectively increasing the reach of safety planning in these catchments). The potential dependence of intervention effects on local context has significant implications for the development of multi-component suicide prevention programmes, as combinations of interventions that are optimal at a state or national level will not necessarily be optimal at a regional level. Our analyses suggest that the impact of independent regional planning (i.e. compared with centralised planning) on suicidal behaviour may be substantial, in some cases approaching that of implementing intensive post-attempt care (see Fig. 1). However, the capacity of suboptimal commissioning decisions to severely limit any potential benefit of local programme development is high; many possible combinations of the interventions modelled here prevent substantially fewer suicides than the optimal state-level combinations in those PHN catchments where optimal regional planning has a significant impact on projected suicide mortality (see Fig. 3).

The potentially substantial regional differences in the effects of individual suicide prevention measures observed in our simulation 


\begin{tabular}{|c|c|c|c|c|c|c|c|c|c|c|c|c|c|c|c|}
\hline Murrumbidgee & 6.8 & 8.1 & 3.2 & 2.2 & 3 & 2.7 & 0.1 & -1 & 4.4 & 0.3 & 0 & 0.2 & 0.9 & 5.2 & 0.4 \\
\hline North Coast & 6.8 & 3.1 & 1.2 & 0.6 & 5.4 & 5.3 & 0.3 & -6.4 & 5.5 & 1.9 & 0 & 0 & 1.3 & 0 & 0 \\
\hline HNECC & 6.8 & 3.3 & 1.3 & 0.6 & 4.4 & 4.2 & 0.1 & -4.6 & 5.1 & 0.4 & 0.1 & 0.6 & 1.3 & 0 & 0 \\
\hline Western NSW & 6.8 & 9.9 & 3.8 & 2.3 & 4.3 & 3.5 & 0.1 & -2.2 & 5.2 & 0.2 & 0 & 0.1 & 1.5 & 6 & 1.2 \\
\hline South Eastern NSW & 6.8 & 3 & 1.2 & 0.7 & 3.6 & 3.1 & 0 & -2.4 & 5 & 0 & 0.4 & 0 & 0.6 & 0 & 2.5 \\
\hline South Western Sydney & 7 & 3.5 & 1.3 & 0.5 & 3.5 & 4.3 & -0.1 & 0.1 & 4.7 & 0.2 & 2.1 & 0 & 1 & 0 & 0 \\
\hline Nepean Blue Mountains & 6.8 & 4.1 & 1.6 & 0.6 & 4.3 & 5.3 & -0.2 & -1.8 & 5.1 & 0.1 & 2.3 & 0 & 0.8 & 0 & 0 \\
\hline Western Sydney & 6.9 & 3.6 & 1.4 & 0.4 & 3.4 & 4.2 & -0.1 & 0.3 & 4.7 & 0.2 & 2.6 & 0.3 & 0.5 & 0 & 0.9 \\
\hline Northern Sydney & 6.8 & 3.4 & 1.3 & 0.4 & 6.7 & 8.3 & -0.1 & 1.4 & 6.1 & 0 & 4.7 & 0.7 & 1.3 & 0 & 0.4 \\
\hline \multirow[t]{2}{*}{ Central and Eastern Sydney } & 6.8 & 4.1 & 1.6 & 0.4 & 5.4 & 6.7 & -0.2 & 0.1 & 5.5 & 0 & 4 & 0 & 0.7 & 0 & 0 \\
\hline & 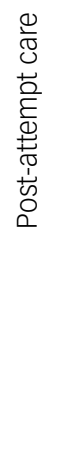 & 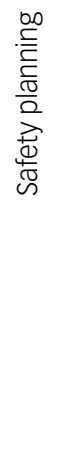 & 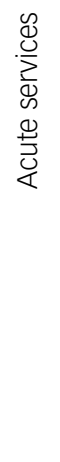 & 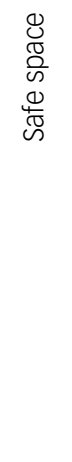 & 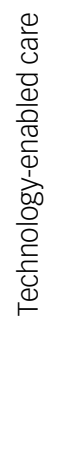 & 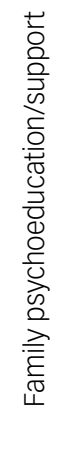 & 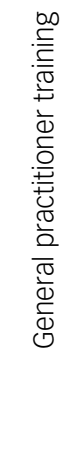 & 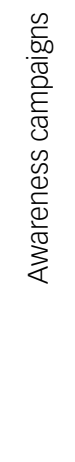 & 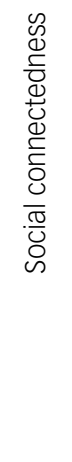 & 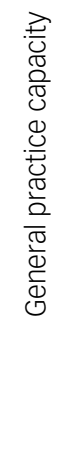 & 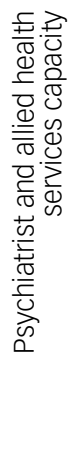 & 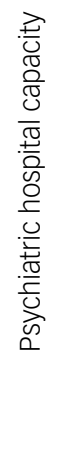 & 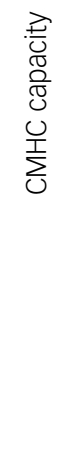 & 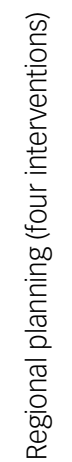 & 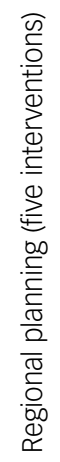 \\
\hline
\end{tabular}

Fig. 1 Percentage reductions in cumulative numbers of suicides over the period 2021-2031 projected under scenarios in which the 13 interventions in Supplementary Table 2 are implemented separately in each Primary Health Network (PHN) catchment (left panel). The panel on the right shows percentage reductions in projected numbers of suicides observed when the optimal combinations of four and five interventions for each PHN catchment are compared with the optimal state-level intervention combinations (combinations of interventions minimising statelevel suicide mortality when implemented in all PHN catchments). Where the optimal combination of interventions at the PHN level is the same as the optimal state-level intervention combination, the percentage reduction in suicide mortality due to regional planning is (necessarily) equal to zero. CMHC, community mental healthcare services; HNECC, Hunter New England and Central Coast; NSW, New South Wales.

analyses (see Fig. 1) are consistent with the results of a previous analysis examining the impacts of selected mental health services interventions on suicide mortality in England and Wales. ${ }^{11}$ While et al. reported significantly greater decreases in suicide rates associated with the implementation of multiple suicide prevention policies in larger mental health services (those reporting higher numbers of patient contacts per year) and services providing care to socioeconomically deprived populations, although the effects of implementing alternative sets of polices in these (and other) services were not considered. ${ }^{11}$ As far as we are aware, the analyses presented

Table 1 Best-performing combinations of interventions at the state and PHN levels (i.e. intervention combinations minimising the number of suicides projected over the period 2021-2031)

$\begin{array}{lll}\text { State-level interventions } & \text { PHN-level interventions } & \text { PHN(s) }\end{array}$

Four interventions

Post-attempt care, technology-enabled care, family 1 Post-attempt care, safety planning, acute care services, social psychoeducation, social connectedness

Five interventions

Post-attempt care, safety planning, technologyenabled care, family psychoeducation, social connectedness

connectedness

1 Post-attempt care, technology-enabled care, family psychoeducation, awareness campaigns, social connectedness

2 Post-attempt care, technology-enabled care, family psychoeducation, Western sydney social connectedness, psychiatrist and allied health services capacity increase

3 Post-attempt care, safety planning, family psychoeducation, Western NSW awareness campaigns, social connectedness

4 Post-attempt care, safety planning, acute care services, technology- Murrumbidgee enabled care, social connectedness 


\section{Four interventions}

$\begin{array}{lll}\text { PHN } & \text { Prevented } & \text { Reduction (95\% interval) } \\ \text { Western NSW } & 19.57 & 5.96(3.50-8.37) \\ \text { Murrumbidgee } & 17.34 & 5.25(3.21-7.23)\end{array}$

Five interventions

$\begin{array}{lll}\text { PHN } & \text { Prevented } & \text { Reduction (95\% interval) } \\ \text { Northern Sydney } & 2.95 & 0.42(-1.52 \text { to } 2.23) \\ \text { Western Sydney } & 7.31 & 0.85(0.14-1.57) \\ \text { South Eastern NSW } & 16.22 & 2.38(0.25-4.03) \\ \text { Western NSW } & 3.49 & 1.17(-0.02 \text { to } 2.29) \\ \text { Murrumbidgee } & 1.18 & 0.39(-0.50 \text { to } 1.13)\end{array}$

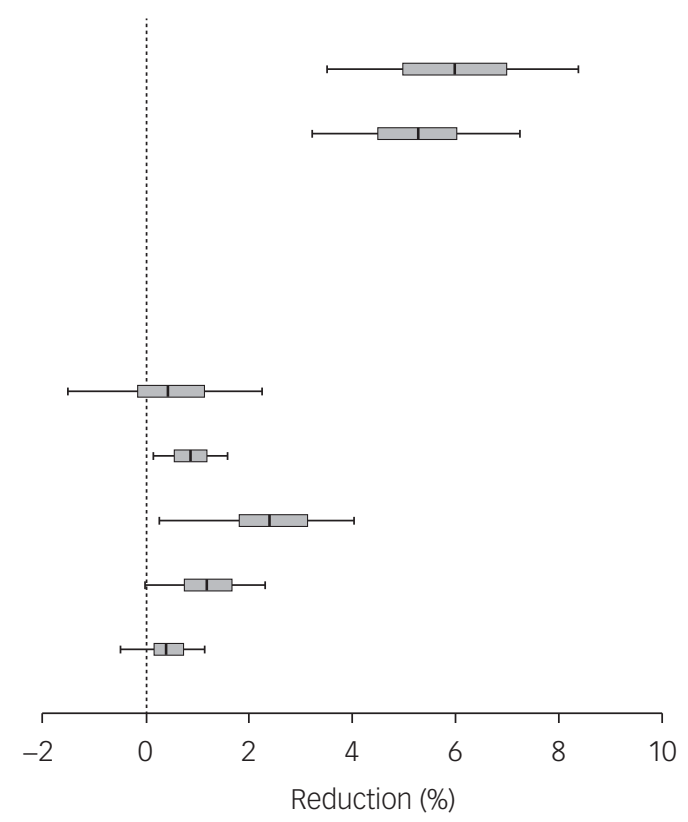

Fig. 2 Projected reductions in total numbers of suicides over the period 2021-2031 observed when the optimal combinations of four and five interventions at the Primary Health Network (PHN) level are compared with the optimal state-level intervention combinations (see percentage reductions and $95 \%$ intervals reported in the rightmost column were derived from the distributions of projected numbers of suicides calculated in the sensitivity analyses. Note that the $95 \%$ intervals provide a measure of the impact of uncertainty in the assumed intervention effects, but should not be interpreted as confidence intervals. Mean percentage reductions and $50 \%$ and $95 \%$ intervals are plotted on the right. NSW, New South Wales.

here are the first to quantify the potential impact of independent regional planning (relative to national- or state-level planning) on the effectiveness of suicide prevention programmes. Our modelling provides conditional support for the Australian Government's decision, made in response to the National Mental Health Commission's 2014 National Review of Mental Health Programmes and Services, to devolve responsibility for the commissioning of suicide prevention services to the (then) recently
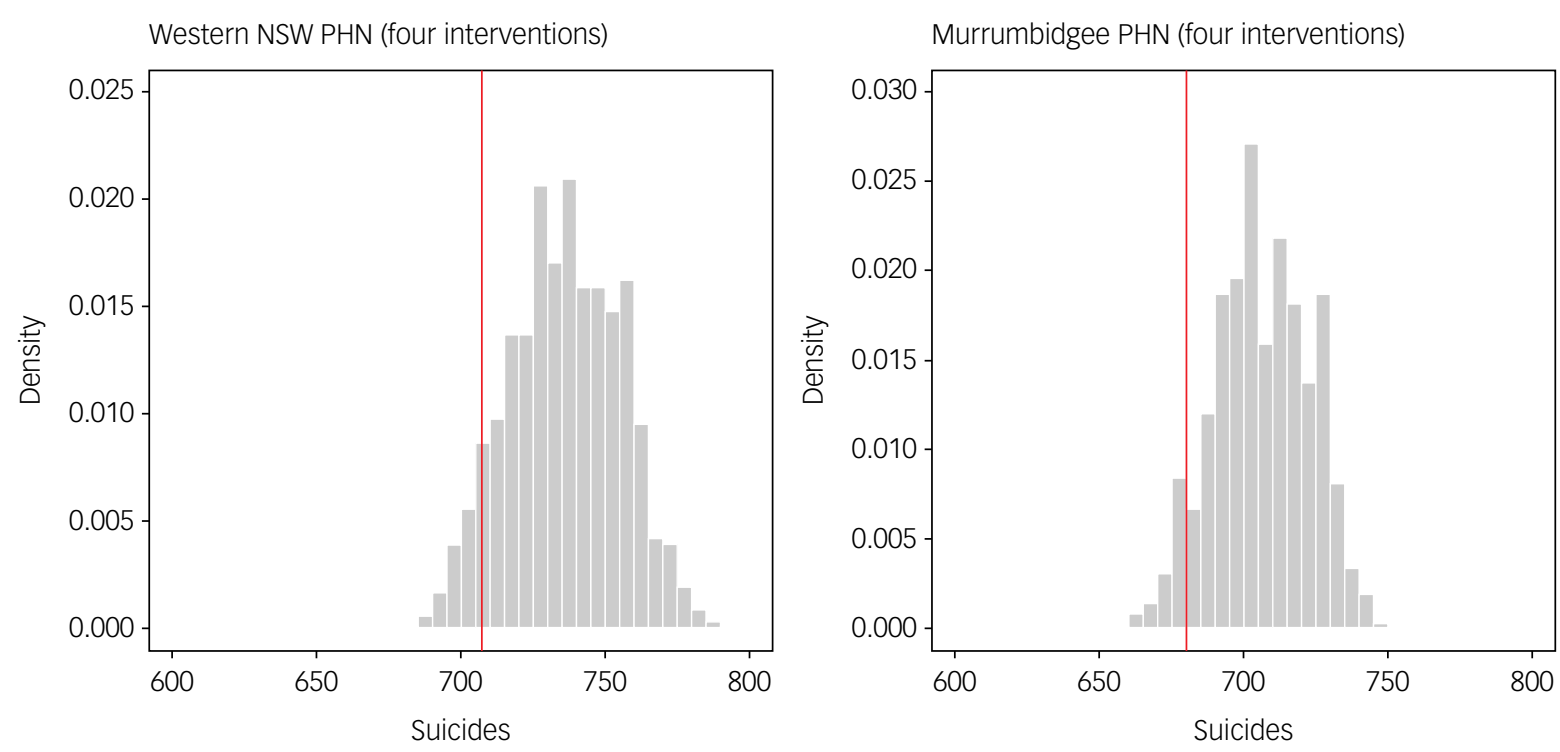

Fig. 3 Distributions of total (cumulative) numbers of suicides projected for the Western New South Wales (NSW) and Murrumbidgee Primary Health Network (PHN) catchments over the period 2011-2031 under all (715) possible combinations of four interventions selected from the 13 interventions in Supplementary Table 2. The red vertical lines correspond to total numbers of suicides projected under the optimal state-level combination of interventions (i.e. the combination of interventions minimising state-level suicide mortality when implemented in all PHN catchments). 
established PHNs. ${ }^{19}$ To the extent that it enables optimal tailoring of multi-component suicide prevention programmes to the specific needs of local communities and services, this devolution of responsibility for suicide prevention planning has the potential to significantly reduce suicidal behaviour at a regional level (see Figs 1 and 2).

Although the transition to regional commissioning of mental health and suicide prevention services in Australia potentially facilitates the development of locally optimal suicide prevention programmes, the results of our analyses indicate that this will require that PHNs are provided with the necessary resources and tools to support reliable, evidence-based decision-making. The National Mental Health Service Planning Framework (NMHSPF) and NMHSPF Planning Support Tool, which originated with Australia's Fourth National Mental Health Plan (2009-2014), were developed to guide regional mental health services planning decisions, providing estimates of future demand for mental healthcare and the resources (mix of service types, workforce, funding, etc.) needed to meet that demand. ${ }^{20}$ More recently, we have applied participatory modelling methods to develop interactive dynamic simulation models designed specifically to support regional commissioning and coordination of suicide prevention services. ${ }^{16,21,22}$ These models provide a logically consistent 'what-if tool that can be used to improve understanding of the possible impacts of proposed initiatives before they are implemented in the real world, and, unlike the NMHSPF and other static models, are capable of capturing highly complex system behaviour resulting from feedback, delayed effects and intervention interactions. ${ }^{23,24}$ Routine use of advanced decision support tools such as these in suicide prevention planning is likely to be critical if the potentially significant reductions in suicide mortality attainable through local programme development are to be realised.

\section{Limitations}

This study has a number of important limitations that should be noted. First, the results presented here depend on the specific suicide prevention and mental health services interventions included in the simulations and the particular distribution of demographic, health and services system characteristics in our study population, so the external validity of our conclusions is potentially limited. Analyses examining the effects of independent regional suicide prevention planning in other contexts (i.e. different interventions and populations) are needed to determine the range of conditions under which local programme development may be expected to contribute to significant decreases in suicide mortality. Second, we have only considered the potential impacts of implementing optimal combinations of suicide prevention and mental health services interventions in each PHN catchment; however, independent local planning may increase the effectiveness of suicide prevention programmes in other ways, including through greater stakeholder engagement and by providing increased opportunity for identifying promising new initiatives and improved approaches to implementation. ${ }^{25,26}$ Potential reductions in suicidal behaviour resulting from a shift to autonomous regional planning may therefore be significantly greater than our modelling suggests. Finally, the simulation analyses presented here effectively disregard the substantial adverse mental health impacts of social dislocation and unemployment resulting from the ongoing COVID-19 pandemic. ${ }^{27,28}$ Nevertheless, preliminary analyses indicate that incorporating the effects of COVID-19 on psychological distress and suicidal behaviour in our simulations would not significantly affect our principal conclusions (see Supplementary Material).

In conclusion, the simulation modelling analyses presented here indicate that the effectiveness of individual suicide prevention measures may depend substantially on the specific populations and services systems in which those measures are implemented, so that evidence-based, multi-component suicide prevention programmes developed at a national or state level will not necessarily be optimal at a regional level. Potentially substantial reductions in local suicide mortality, comparable to those projected for effective clinical interventions (e.g. post-attempt care; see Fig. 1), may be achievable by devolving responsibility for the commissioning of mental health and suicide prevention services implemented as part of a national suicide prevention strategy to regional health authorities. Nevertheless, our results also indicate that the capacity of suboptimal commissioning decisions to limit any potential benefit of local programme development is high, emphasising the need to ensure that regional planners are provided with the resources and tools required to support reliable, evidence-based decision-making. Dynamic simulation models, co-developed with local stakeholders using participatory modelling methods, offer perhaps the most promising means of addressing the challenge of developing regional suicide prevention programmes that are consistent with the best available scientific evidence while also accommodating heterogeneity in local services and community needs.

Adam Skinner (D), Brain and Mind Centre, Faculty of Medicine and Health, University of Sydney, Australia; Jo-An Occhipinti, Brain and Mind Centre, Faculty of Medicine and Health, University of Sydney, Australia; and Computer Simulation and Advanced

Research Technologies, Australia; Yun Ju Christine Song, Brain and Mind Centre,

Faculty of Medicine and Health, University of Sydney, Australia; Ian B. Hickie, Brain and Mind Centre, Faculty of Medicine and Health, University of Sydney, Australia

Correspondence: Adam Skinner. Email: adam.skinner@sydney.edu.au

First received 28 Mar 2021, final revision 29 Jun 2021, accepted 23 Jul 2021

\section{Supplementary material}

Supplementary material is available online at https://doi.org/10.1192/bjo.2021.989

\section{Data availability}

The authors confirm that the data supporting the findings of this study are available within the article and its supplementary materials. Details of all data sources used for model parameterisation and calibration are provided in the Supplementary Materia

\section{Acknowledgements}

We are grateful to members of the National Mental Health Systems Modelling Network for helpful discussion.

\section{Author contributions}

A.S., J.-A.O., Y.J.C.S. and I.B.H. conceived the study. A.S. built the system dynamics model, performed the analyses and drafted the paper. J.-A.O., Y.J.C.S. and I.B.H. contributed to preparation of the final manuscript. The corresponding author attests that all listed authors meet authorship criteria and that no others meeting the criteria have been omitted. All authors had full access to the study data and final responsibility for the decision to submit for publication.

\section{Funding}

The research presented in this article was funded by a National Health and Medical Research Council (NHMRC) Centres of Research Excellence grant (application identifier 1171910). I.B.H. is supported by an NHMRC Research Fellowship (application identifier 1136259). The NHMRC had no role in study design, data analysis, interpretation of the results or preparation of the manuscript.

\section{Declaration of interest}

J.-A.O. is Head of Systems Modelling, Simulation \& Data Science at the Brain and Mind Centre University of Sydney and Managing Director of Computer Simulation and Advanced Research Technologies (CSART)

B.H. was an inaugural Commissioner on Australia's National Mental Health Commission (2012-2018). He is the Co-Director, Health and Policy at the Brain and Mind Centre, University of Sydney. The Brain and Mind Centre operates an early-intervention youth service at Camperdown under contract to headspace. I.B.H. has previously led community-based and pharmaceutical industry-supported (Wyeth, Eli Lily, Servier, Pfizer, AstraZeneca) projects focused on the identification and better management of anxiety and depression; was a member of the Medical Advisory Panel for Medibank Private until October 2017, a Board Member of Psychosis Australia Trust and a member of Veterans Mental Health Clinical Reference group; 
and is the Chief Scientific Advisor to, and a 5\% equity shareholder in, InnoWell Pty Ltd. InnoWell was formed by the University of Sydney (45\% equity) and PwC (Australia; $45 \%$ equity) to deliver the $\$ 30$ million Australian Government-funded Project Synergy (2017-2020; a 3-year programme for the transformation of mental health services) and to lead transformation of mental health services internationally through the use of innovative technologies. A.S. and Y.J.C.S. have no competing interests to declare.

\section{References}

1 Naghavi M. Global, regional, and national burden of suicide mortality 1990 to 2016: systematic analysis for the Global Burden of Disease Study 2016. BMJ 2019; 364: 194.

2 World Health Organization. Global Health Estimates 2016: Deaths by Cause, Age, Sex, by Country and by Region, 2000-2016. World Health Organization 2018 (https://www.who.int/data/global-health-estimates).

3 World Health Organization. Mental Health Action Plan 2013-2020. World Health Organization, 2013 (https://www.who.int/publications/i/item/9789241506021).

4 World Health Organization. National Suicide Prevention Strategies: Progress, Examples and Indicators. World Health Organization, 2018 (https://www. who.int/publications/i/item/national-suicide-prevention-strategies-progressexamples-and-indicators).

5 Middleton N, Sterne JAC, Gunnell D. The geography of despair among 15-44year-old men in England and Wales: putting suicide on the map. J Epidemiol Community Health 2006; 60: 1040-7.

6 Pirkola S, Sund R, Sailas E, Wahlbeck K. Community mental-health services and suicide rate in Finland: a nationwide small-area analysis. Lancet 2009; 373 : 147-53.

7 Cheung YTD, Spittal MJ, Pirkis J, Yip PSF. Spatial analysis of suicide mortality in Australia: investigation of metropolitan-rural-remote differentials of suicide risk across states/territories. Soc Sci Med 2012; 75: 1460-8.

8 Phillips JA. Factors associated with temporal and spatial patterns in suicide rates across U.S. States, 1976-2000. Demography 2013; 50: 591-614.

9 Chang S, Sterne JAC, Wheeler BW, Lu T, Lin J, Gunnell D. Geography of suicide in Taiwan: spatial patterning and socioeconomic correlates. Health Place 2011; 17: $641-50$

10 Hsu C, Chang S, Lee EST, Yip PSF. Geography of suicide in Hong Kong: spatia patterning and socioeconomic correlates and inequalities. Soc Sci Med 2015 130: 190-203.

11 While $\mathrm{D}$, Bickley $\mathrm{H}$, Roscoe $\mathrm{A}$, Windfuhr $\mathrm{K}$, Rahman $\mathrm{S}$, Shaw J, et at. Implementation of mental health service recommendations in England and Wales and suicide rates, 1997-2006: a cross-sectional and before-and-after observational study. Lancet 2012; 379: 1005-12.

12 Kapur N, Ibrahim S, While D, Baird A, Rodway C, Hunt IM, et al. Mental health service changes, organisational factors, and patient suicide in England in 1997-2012: a before-and-after study. Lancet Psychiatry 2016; 3: 526-34.

13 Appleby L, Hunt IM, Kapur N. New policy and evidence on suicide prevention. Lancet Psychiatry 2017; 4: 658-60.

14 Australian Bureau of Statistics. National, State and Territory Population, Mar 2020. Cat. No. 3101.0. Australian Bureau of Statistics, 2020 (https://www. abs.gov.au/statistics/people/population/national-state-and-territory-population/latest-release).

15 Australian Bureau of Statistics. Technical Paper Socio-Economic Indexes for Areas (SEIFA), 2016. Cat. No. 2033.0.55.001. Australian Bureau of Statistics, 2018 (https://www.ausstats.abs.gov.au/ausstats/subscriber.nsf/0/756EE3DBE FA869EFCA258259000BA746/\$File/SEIFA\%202016\%20Technical\%20Paper.pdf).

16 Occhipinti J, Skinner A, Carter S, Health J, Lawson K, McGill K, et al. Federal and state cooperation necessary but not sufficient for effective regional mental health systems: insights from systems modelling and simulation. Sci Rep 2021; 11: 11209

17 Sterman JD. Business Dynamics: Systems Thinking and Modeling for a Complex World. McGraw-Hill, 2000

18 Powell MJD. The BOBYQA Algorithm for Bound Constrained Optimization with out Derivatives. Technical Report No. DAMTP 2009/NA06. Department of Applied Mathematics and Theoretical Physics, Cambridge University, 2009 (http://www.damtp.cam.ac.uk/user/na/NA_papers/NA2009_06.pdf).

19 Hickie I. Putting mental health services and suicide prevention reform into practice. Public Health Res Pract 2017; 27: e2721710.

20 The University of Queensland. Introduction to the National Mental Health Services Planning Framework - Commissioned by the Australian Government Department of Health. Version AUS V2.2. The University of Queensland, 2019 (https://qcmhr.org/wp-content/uploads/2021/01/Introduction-to-the-NMHSPFAUS-V2.2.pdf).

21 Page A, Atkinson J, Campos W, Heffernan M, Ferdousi S, Power A, et al. A decision support tool to inform local suicide prevention activity in Greater Western Sydney (Australia). Aust N Z J Psychiatry 2018; 52: 983-93.

22 Atkinson J, Skinner A, Hackney S, Mason L, Heffernan M, Currier D, et al. Systems modelling and simulation to inform strategic decision making for suicide prevention in rural New South Wales (Australia). Aust NZ J Psychiatry 2020; 54: 892-901.

23 Homer JB, Hirsch GB. System dynamics modeling for public health: background and opportunities. Am J Public Health 2006; 96: 452-8.

24 Sterman JD. Learning from evidence in a complex world. Am J Public Health 2006; 96: 505-14.

25 Headey A, Pirkis J, Merner B, VandenHeuval A, Mitchell P, Robinson J, et al. A review of 156 local projects funded under Australia's National Suicide Prevention Strategy: overview and lessons learned. AeJAMH 2006; 5: 247-61.

26 Hickie IB. Time to implement national mental health reform. Med J Aust 2015 202: 515-8.

27 Moreno C, Wykes T, Galderisi S, Nordentoft M, Crossley N, Jones N, et al. How mental health care should change as a consequence of the COVID-19 pandemic. Lancet Psychiatry 2020; 7: 813-24.

28 Atkinson J, Song YJC, Merikangas KR, Skinner A, Prodan A, lorfino F, et al. The science of complex systems is needed to ameliorate the impacts of COVID-19 on mental health. Front Psychiatry 2020; 11: 606035. 